\title{
Depressive and anxiety symptoms and cortical amyloid deposition among cognitively normal elderly persons: the Mayo Clinic Study of Aging
}

\author{
Janina Krell-Roesch, ${ }^{1}$ Val J. Lowe, ${ }^{2}$ Jennifer Neureiter, ${ }^{3 *}$ Anna Pink, ${ }^{1,3}$ \\ Rosebud O. Roberts, ${ }^{4,5}$ Michelle M. Mielke, ${ }^{4,5}$ Prashanthi Vemuri, ${ }^{2}$ \\ Gorazd B. Stokin, ${ }^{6}$ Teresa J. Christianson, ${ }^{4}$ Clifford R. Jack, Jr., ${ }^{2}$ David S. Knopman, ${ }^{5}$ \\ Bradley F. Boeve, ${ }^{5}$ Walter K. Kremers, ${ }^{4}$ Ronald C. Petersen ${ }^{4,5}$ and Yonas E. Geda ${ }^{1,5,7,8}$ \\ ${ }^{1}$ Translational neuroscience and Aging Program, Mayo Clinic, Scottsdale, Arizona, USA \\ ${ }^{2}$ Department of Radiology, Mayo Clinic, Rochester, Minnesota, USA \\ ${ }^{3}$ Paracelsus Medical University, Salzburg, Austria \\ ${ }^{4}$ Department of Health Sciences Research, Mayo Clinic, Rochester, Minnesota, USA \\ ${ }^{5}$ Department of Neurology, Mayo Clinic, Rochester, Minnesota, USA \\ ${ }^{6}$ International Clinical Research Center, St. Anne's University Hospital, Brno, Czech Republic \\ ${ }^{7}$ Department of Psychiatry and Psychology, Mayo Clinic, Scottsdale, Arizona, USA \\ ${ }^{8}$ Department of Neurology, Mayo Clinic, Scottsdale, Arizona, USA
}

Background: Little is known about the association of cortical $\mathrm{A} \beta$ with depression and anxiety among cognitively normal (CN) elderly persons.

Methods: We conducted a cross-sectional study derived from the population-based Mayo Clinic Study of Aging in Olmsted County, Minnesota; involving CN persons aged $\geq 60$ years that underwent PiB-PET scans and completed Beck Depression Inventory-II (BDI-II) and Beck Anxiety Inventory (BAI). Cognitive diagnosis was made by an expert consensus panel. Participants were classified as having abnormal ( $\geq 1.4$; $\mathrm{PiB}+$ ) or normal PiB-PET $(<1.4 ; \mathrm{PiB}-)$ using a global cortical to cerebellar ratio. Multi-variable logistic regression analyses were performed to calculate odds ratios (OR) and 95\% confidence intervals (95\% CI) after adjusting for age and sex.

Results: Of 1,038 CN participants (53.1\% males), 379 were $\mathrm{PiB}+$. Each one point symptom increase in the $\mathrm{BDI}(\mathrm{OR}=1.03 ; 1.00-1.06)$ and $\mathrm{BAI}(\mathrm{OR}=1.04 ; 1.01-1.08)$ was associated with increased odds of PiBPET + . The number of participants with BDI $>13$ (clinical depression) was greater in the PiB-PET + than PiB-PET- group but the difference was not significant $(\mathrm{OR}=1.42 ; 0.83-2.43)$. Similarly, the number of participants with BAI $>10$ (clinical anxiety) was greater in the PiB-PET + than PiB-PET - group but the difference was not significant $(\mathrm{OR}=1.77 ; 0.97-3.22)$.

Conclusions: As expected, depression and anxiety levels were low in this community-dwelling sample, which likely reduced our statistical power. However, we observed an informative albeit weak association between increased BDI and BAI scores and elevated cortical amyloid deposition. This observation needs to be tested in a longitudinal cohort study.

Key words: depressive symptoms, anxiety symptoms, cortical amyloid deposition, cognitively normal persons

\section{Introduction}

${ }^{*}$ Dr. Neureiter was a medical student when she did her research thesis work under the mentorship of Professor Geda. She is now doing residency training at Paracelsus Medical University, Salzburg, Austria.

Correspondence should be addressed to: Yonas E. Geda, MD, MSc, Professor of Neurology and Psychiatry, Consultant, Department of Psychiatry \& Psychology and Department of Neurology, Mayo Clinic, 13400 East Shea Boulevard, Scottsdale, AZ 85259, Arizona. Phone: +480-301-4343; Fax: +480-301-7017. E-mail: geda.yonas@mayo.edu. Received 10 Feb 2017; revision requested 28 Feb 2017; revised version received 28 May 2017; accepted 30 Sep 2017. First published online 4 December 2017.
Alzheimer's disease (AD) pathology likely precedes the development of clinical manifestations by several years or decades (Braak and Braak, 1991; Shaw et al., 2007). In order to study preclinical $\mathrm{AD}$, it is critical to use in vivo biomarkers such as amyloid imaging (Dubois et al., 2007; Sperling et al., 2011). The amyloid cascade hypothesis posits that the accumulation of amyloid $\beta$-peptide in the brain is a necessary component of the 
pathophysiology of AD (Hardy and Selkoe, 2002; Choi et al., 2014). About $30 \%$ of cognitively normal persons above the age of 70 years, $60 \%$ of patients with mild cognitive impairment (MCI) and $85 \%$ of clinically diagnosed $\mathrm{AD}$ patients are amyloid positive (Jack et al., 2013). However, studies have found an association between amyloid deposition with a faster cognitive decline in both cognitively normal elderly (Morris et al., 2009; Chetelat et al., 2012; Knopman et al., 2012; Petersen et al., 2016) and MCI subjects (Jack et al., 2010; Jagust et al., 2010). A growing body of research has shown that depressive and anxiety symptoms may increase the risk of cognitive decline (Geda et al., 2006, 2008, 2014; Pink et al., 2015). Furthermore, we and others have reported that depressive (Donovan et al., 2015; Krell-Roesch et al., 2016) and anxiety symptoms (Pink et al., 2017) are associated with neuroimaging biomarkers of AD, specifically FDG-PET and cortical thickness as measured by MRI, among cognitively normal elderly participants. To date, only few studies have investigated the association between amyloid imaging and depression (Butters et al., 2008; Chung et al., 2016; Holmes et al., 2016; Harrington et al., 2017) or anxiety (Pietrzak et al., 2014; Pietrzak et al., 2015; Holmes et al., 2016). We therefore sought to examine the association between cortical amyloid deposition, as measured by Pittsburgh compound B positron emission tomography (PiB-PET), depressive and anxiety symptoms in a population-based sample of cognitively normal participants. Given the current discussion in the literature on whether prodromal neuropsychiatric symptoms should be assessed on a continuum rather than relying on arbitrary cut-offs (Altman and Royston, 2006; Bjelland et al., 2009; Laborde-Lahoz et al., 2014), we used both continuous and categorical approaches when assessing depressive and anxiety symptoms. We hypothesized that both anxiety and depressive symptoms would be associated with higher cortical amyloid deposition as measured by PiB-PET in our sample of community-dwelling cognitively normal elderly persons.

\section{Methods}

Setting. This study was conducted in the setting of the population-based Mayo Clinic Study of Aging (MCSA). Details of the design and conduct of the MCSA have been reported elsewhere (Roberts et al., 2008). Briefly, the MCSA is an ongoing population-based study of cognitive aging in Olmsted County, Minnesota. Participants who were $\geq 60$ years old, had undergone brain amyloid imaging and completed both depressive and anxiety symptom assessments were included in this study. The study was conducted with the approval of the institutional review boards of the Mayo Clinic and Olmsted Medical Center in Rochester, Minnesota and with the written informed consent of each participant.

Cognitive evaluation. Participants underwent extensive evaluation including neurological examination, psychometric testing and risk factor assessment as described in detail elsewhere (Roberts et al., 2008). An expert consensus panel consisting of physicians, neuropsychologists, and nurses or study coordinators reviewed all pertinent data and made the diagnosis of normal cognition, MCI, or dementia. Individuals were considered cognitively normal at baseline according to published normative data developed on this community (Ivnik et al., 1992a,1992b, 1992c, 1992d; Malec et al., 1992). For MCI, the following revised Mayo Clinic criteria for MCI (Petersen, 2004; Winblad et al., 2004) were used: (1) cognitive concern expressed by a physician, informant, participant, or nurse; (2) impairment in one or more cognitive domains (memory, language, visuospatial skills, or executive functions); (3) essentially normal functional activities; and (4) absence of dementia. Participants with MCI had a Clinical Dementia Rating Scale score of 0 or 0.5 ; however, the final diagnosis of MCI was based on all available data. Participants with MCI or dementia were excluded from this study.

Measurement of depressive and anxiety symptoms. Depressive symptoms were measured using a validated, self-administered Beck Depressive Inventory-II (BDI) which is a sensitive instrument in elderly subjects (Beck et al., 1996). Thus, the BDI-II likely captures as many depressive symptoms as possible and we considered it to be an appropriate screening instrument to use in a large scale population-based study. The BDI-II consists of 21 items such as feeling guilty and loss of interest, that are assessed over the last two weeks and are rated in severity on a 4-point Likert scale ranging from 0 to 3 ; the total score ranges from 0 to 63. Symptoms of anxiety were measured using the Beck Anxiety Inventory (BAI), a validated, self-administered questionnaire (Beck et al., 1988). Like the BDI-II, the BAI also consists of 21 items that are assessed over the last week and measure common symptoms of anxiety, such as nervousness and fear of losing control. The severity of each symptom is rated ranging from 0 to 3 with a total score ranging from 0 to 63 .

Neuroimaging of amyloid deposition. We performed amyloid PET imaging using Pittsburgh Compound B tracer. PiB scans, consisting of four 
5-minute dynamic frames, were acquired from 40 to 60 minutes after intravenous injection with 292-728 MBq $\left({ }^{11} \mathrm{C}\right) \mathrm{PiB}$. Images were analyzed using an in-house, fully automated image processing pipeline, in which image voxel values were extracted from automatically labeled regions of interest propagated from an MRI template. A global amyloid PET standardized uptake value ratio (SUVR) was formed from the prefrontal, orbitofrontal, parietal, temporal, anterior cingulate, and posterior cingulate/precuneus regions of interest normalized to the cerebellar grey matter. Participants with an SUVR $\geq 1.40$ were classified as having an abnormal PiB-PET (amyloid positive; $\mathrm{PiB}+$ ) as we have previously validated by autopsy (Murray et al., 2015).

Statistical Analysis. We used multi-variable logistic regression analyses to examine the association between BDI and BAI scores with an abnormal PiB-PET scan (PiB+ vs. PiB-, modeled on BAI, $\mathrm{BDI}$ ) by calculating odds ratios (OR) and 95\% confidence intervals $(95 \% \mathrm{CI})$ after adjusting for age and sex. Furthermore, we conducted linear regression analyses to investigate the association between BDI and BAI scores with elevated amyloid deposition in the brain (PiB amyloid SUVR modeled on BAI, BDI) by computing $\beta$ estimates, and standard errors of the mean (SE) after adjusting for age and sex. For both regression analyses, we treated both BDI-II and BAI as continuous variables. In addition, we examined BDI-II and BAI as categorical variables with different cut-offs (BDI-II cut-offs: 4 (median number of symptoms in the study population), and 13 (cut-off for clinical symptoms); BAI cutoffs: 2 (median number of symptoms in the study population), and 10 (cut-off for clinical symptoms). Furthermore, we conducted an analysis stratified by four quartiles (with no symptoms being the reference group; score $=0$ ). Statistical testing was done at the conventional 2-tailed alpha level of $p<0.05$. All analyses were performed using SAS System, version 9.3 software (SAS Institute, Cary, NC).

\section{Results}

Of 1,038 cognitively normal participants, 551 $(53.1 \%)$ were males and the median age was 73 years (interquartile range, IQR 67, 79). Three hundred and seventy nine participants $(37 \%)$ were classified as having an abnormal amyloid deposition $(\mathrm{PiB}+) . \mathrm{PiB}+$ participants were significantly older than $\mathrm{PiB}-$ participants $(p<0.001)$. In addition, $39 \%$ of $\mathrm{PiB}+$ participants were APOE $\varepsilon 4$ carriers whereas only $19.9 \%$ of $\mathrm{PiB}-$ participants were
APOE $\varepsilon 4$ carriers $(p<0.001)$. There were few participants with clinical depression $(N=63$, $6.1 \%)$ or clinical anxiety $(N=51,4.9 \%)$, and the median BDI (3; IQR 1, 6) and BAI $(1$; IQR 0, 4) scores were low. Table 1 shows the demographic characteristics of $\mathrm{PiB}+, \mathrm{PiB}-$ and total study population. In all regression analyses, the predictor variables $(\mathrm{PiB}+$ vs. $\mathrm{PiB}-$ as categorical variables; and $\mathrm{PiB}$ amyloid SUVR as continuous variable) were modeled on the outcome variables (BAI and $\mathrm{BDI}$ as categorical and continuous variables). The logistic regression analysis, adjusted for age and sex, revealed that both depression $(\mathrm{OR}=1.03$; $1.00-1.06)$ and anxiety ( $\mathrm{OR}=1.04 ; 1.01-1.08)$ were associated with higher odds for an abnormal PiB-PET when examined as continuous variables. The association was significant for anxiety $(p=$ $0.022)$ and marginally significant for depression $(p=0.077)$. When we categorized BDI-II and BAI, we observed that the odds of being PiBPET+ were elevated for participants with BDI $\geq$ 13 which indicates clinical depression as compared to participants with $\mathrm{BDI}<13(\mathrm{OR}=1.42 ; 0.83-$ 2.43). The same pattern was found for participants with $\mathrm{BAI} \geq 10$ which indicates clinical anxiety as compared to participants with BAI $<10$ (OR = $1.77 ; 0.97-3.22)$. The effect sizes were smaller for the other group comparisons (BDI: $\geq 10$ vs. $<10$, $\geq 4$ vs. $<4$; BAI: $\geq 8$ vs. $<8, \geq 2$ vs. $<2$ ). None of these associations reached statistical significance. The stratified analysis by quartiles also showed a trend for higher odds with higher scores on BDI and BAI as compared to the reference groups (score $=0$; no symptoms), however lacking statistical significance (Table 2). When we examined the association between amyloid deposition as measured by SUVR and depressive and anxiety symptoms (both treated as numeric variables) using linear regression analysis, we observed significant associations between depressive symptoms $(\beta=0.005, \mathrm{SE}=$ $0.002, p=0.007)$ and amyloid deposition as well as between anxiety symptoms $(\beta=0.006, \mathrm{SE}=$ 0.003, $p=0.027$ ) and amyloid deposition, after adjusting for age and sex (Table 3 ).

\section{Discussion}

Here, we report the cross-sectional association between amyloid deposition as measured by PiBPET and depressive and anxiety symptoms among community-dwelling persons free of MCI or dementia. We and others have examined the role of neuropsychiatric symptoms in predicting the progression from $\mathrm{CN}$ status to incident MCI (Geda et al., 2014). Furthermore, an expert panel of the Alzheimer's Association (ISTAART 
Table 1. Demographics characteristics of study participants

\begin{tabular}{|c|c|c|c|c|}
\hline & $\begin{array}{l}\mathrm{PIB}+(\geq 1.4) \\
(\mathrm{N}=379) \\
\mathrm{N}(\%)\end{array}$ & $\begin{array}{l}\mathrm{PIB}-(<1.4) \\
(\mathrm{N}=659) \\
\mathrm{N}(\%)\end{array}$ & $\begin{array}{l}\text { TотAL } \\
(\mathrm{N}=1,038) \\
\mathrm{N}(\%)\end{array}$ & $p$ VALUE \\
\hline Male & $196(51.7)$ & $355(53.9)$ & $551(53.1)$ & $0.503^{1}$ \\
\hline \multicolumn{5}{|l|}{ Age (years) } \\
\hline Median (IQR) & $77(71,82)$ & $72(65,77)$ & $73(67,79)$ & \multirow[t]{3}{*}{$<0.001^{2}$} \\
\hline $60-74$ & $153(40.4)$ & $414(62.8)$ & $567(54.6)$ & \\
\hline $75-95$ & $226(59.6)$ & $245(37.2)$ & $471(45.4)$ & \\
\hline \multicolumn{5}{|l|}{ Education (years) } \\
\hline Median (IQR) & $14(12,16)$ & $15(12,17)$ & $14(12,17)$ & $0.090^{2}$ \\
\hline$>12$ Years & $263(69.4)$ & $482(73.1)$ & $745(71.8)$ & $0.196^{1}$ \\
\hline \multicolumn{5}{|l|}{ BAI total $(0-63)$} \\
\hline Median (IQR) & $1(0,4)$ & $1(0,3)$ & $1(0,4)$ & $0.112^{2}$ \\
\hline$\geq 10$, anxiety & $24(6.3)$ & $27(4.1)$ & $51(4.9)$ & $0.109^{1}$ \\
\hline$\geq 8$ & $38(10.0)$ & $52(7.9)$ & $90(8.7)$ & $0.239^{1}$ \\
\hline$\geq 2$, median & $166(43.8)$ & $269(40.8)$ & $435(41.9)$ & $0.349^{1}$ \\
\hline Q1: 0 & $156(41.2)$ & $297(45.1)$ & $453(43.6)$ & \\
\hline Q2: 1-2 & $84(22.2)$ & $157(23.8)$ & $241(23.2)$ & \\
\hline Q3: 3-5 & $75(19.8)$ & $116(17.6)$ & $191(18.4)$ & \\
\hline Q4: 6-36 & $64(16.9)$ & $89(13.5)$ & $153(14.7)$ & \\
\hline \multicolumn{5}{|l|}{ BDI-II total (0-63) } \\
\hline Median (IQR) & $3(1,7)$ & $3(1,6)$ & $3(1,6)$ & $0.005^{2}$ \\
\hline$\geq 13$, depression & $27(7.1)$ & $36(5.5)$ & $63(6.1)$ & $0.280^{1}$ \\
\hline$\geq 10$ & $55(14.5)$ & $70(10.6)$ & $125(12.0)$ & $0.064^{1}$ \\
\hline$\geq 4$, median & $187(49.3)$ & $274(41.6)$ & $461(44.4)$ & $0.015^{1}$ \\
\hline$\overline{\mathrm{Q}} 1: 0$ & $74(19.5)$ & $155(23.5)$ & $229(22.1)$ & \\
\hline Q2: 1-2 & $81(21.4)$ & $165(25.0)$ & $246(23.7)$ & \\
\hline Q3: 3-6 & $123(32.5)$ & $202(30.7)$ & $325(31.3)$ & \\
\hline Q4: 7-32 & $101(26.6)$ & $137(20.8)$ & $238(22.9)$ & \\
\hline \multicolumn{5}{|c|}{ PiB ratio, PVC (GMWM) } \\
\hline Median (IQR) & $1.63(1.45,1.98)$ & $1.31(1.27,1.34)$ & $1.35(1.29,1.48)$ & $<0.001^{2}$ \\
\hline $\mathrm{APOE} \varepsilon 4$ carrier $^{3}$ & $147(39.0)$ & $130(19.9)$ & $277(26.9)$ & $<0.001^{1}$ \\
\hline Antidepressants & $66(17.4)$ & $130(19.7)$ & $196(18.9)$ & $0.359^{1}$ \\
\hline
\end{tabular}

$\mathrm{IQR}$ = interquartile range; BAI = Beck Anxiety Inventory; BDI = Beck Depression Inventory; PVC = partial volume correction; GMWM $=$ gray matter-white matter; APOE $\varepsilon 4=$ Apolipoprotein $\varepsilon 4$.

${ }^{1} \chi^{2}$ test.

${ }^{2}$ Wilcoxon rank-sum test.

${ }^{3}$ Data missing for seven participants. Antidepressant medication includes SSRI, SNRI, Tetracyclic, Tricyclic, and any other.

Neuropsychiatric Symptoms Professional Interest Area) has also called for the systematic investigation of emotional behavior among cognitively normal persons (Ismail et al., 2016). When we treated depressive and anxiety symptoms as continuous variables, we observed an association in the logistic regression analysis between anxiety and an abnormal PiB-PET as well as depression and an abnormal PiB-PET, even though the latter was not significant. When we assessed depressive and anxiety symptoms as categorical variables, we observed higher effect sizes with higher scores on BDI and BAI. The highest effect sizes were found when we used the clinical cut-offs (BDI $\geq 13$, BAI $\geq 10$ ). The lack of statistical significance for this analysis may be due to low power as only a small percentage of the $\mathrm{PiB}+$ study population reported depression $(7.1 \%)$ and anxiety symptoms $(6.3 \%)$. Similarly, when the associations were stratified by quartiles of BDI-II and BAI symptoms, we observed the same trend: participants in quartiles reflecting higher scores of depressive and anxiety symptoms had higher odds for an abnormal PiB-PET. However, none of these trends reached statistical significance. The results of the linear regression analysis were in line with the logistic regression analysis and revealed a significant association between depressive and anxiety symptoms with amyloid deposition. We also conducted logistic and linear regression analyses stratified by sex and APOE $\varepsilon 4$ carrier status in order to examine potential effects on the association between depression, anxiety and PiB-PET positivity. Neither sex nor APOE $\varepsilon 4$ carrier status significantly altered the results (data not shown). To date, only few studies have examined the cross- 
Table 2. Logistic regression analysis on the association between amyloid positivity with depressive and anxiety symptoms, adjusted for age and sex

\begin{tabular}{|c|c|c|}
\hline & OR $(95 \% \mathrm{CI})$ & $p$ Value \\
\hline BAI total (numeric) & $1.04(1.01,1.08)$ & 0.022 \\
\hline$\geq 10($ vs. $<10)$ & $1.77(0.97,3.22)$ & 0.062 \\
\hline$\geq 8($ vs. $<8)$ & $1.38(0.87,2.19)$ & 0.17 \\
\hline$\geq 2$, median (vs. $<2$ ) & $1.14(0.87,1.49)$ & 0.35 \\
\hline Q1: 0 & (reference) & $0.060^{\mathrm{T}}$ \\
\hline Q2: 1-2 & $1.02(0.72,1.43)$ & 0.93 \\
\hline Q3: 3-5 & $1.27(0.88,1.83)$ & 0.20 \\
\hline Q4: 6-36 & $1.40(0.94,2.08)$ & 0.096 \\
\hline BDI-II total (numeric) & $1.03(1.00,1.06)$ & 0.077 \\
\hline$\geq 13($ vs. $<13)$ & $1.42(0.83,2.43)$ & 0.20 \\
\hline$\geq 10($ vs. $<10)$ & $1.42(0.95,2.11)$ & 0.084 \\
\hline$\geq 4$, median (vs. $<4$ ) & $1.29(0.99,1.68)$ & 0.058 \\
\hline $\bar{Q} 1: 0$ & (reference) & $0.046^{\mathrm{T}}$ \\
\hline Q2: 1-2 & $1.11(0.75,1.66)$ & 0.60 \\
\hline Q3: 3-6 & $1.28(0.88,1.86)$ & 0.19 \\
\hline Q4: 7-32 & $1.45(0.98,2.14)$ & 0.066 \\
\hline
\end{tabular}

$\mathrm{BAI}=$ Beck Anxiety Inventory; BDI $=$ Beck Depression Inventory; $\mathrm{Q}=$ quartile; $\mathrm{T}=$ Trend; $\mathrm{OR}=$ Odds ratio; $95 \% \mathrm{CI}=$ $95 \%$ confidence interval.

Table 3. Linear regression analysis on the association between amyloid SUVR with depressive and anxiety symptoms, adjusted for age and sex

\begin{tabular}{|c|c|c|}
\hline & $\beta \operatorname{EsT}(\mathrm{SE})$ & $p$ VALUE \\
\hline BAI total (continuous) & $0.006(0.003)$ & 0.027 \\
\hline$\geq 10($ vs. $<10)$ & $0.057(0.043)$ & 0.19 \\
\hline$\geq 8$ (vs. $<8)$ & $0.040(0.033)$ & 0.23 \\
\hline$\geq 2$, median (vs. $<2$ ) & $0.016(0.019)$ & 0.39 \\
\hline Q1: 0 & (reference) & $0.066^{\mathrm{T}}$ \\
\hline Q2: 1-2 & $0.014(0.024)$ & 0.55 \\
\hline Q3: 3-5 & $0.035(0.026)$ & 0.18 \\
\hline Q4: 6-36 & $0.045(0.028)$ & 0.11 \\
\hline BDI-II total (continuous) & $0.005(0.002)$ & 0.007 \\
\hline$\geq 13($ vs. $<13)$ & $0.057(0.039)$ & 0.15 \\
\hline$\geq 10$ (vs. $<10)$ & $0.061(0.028)$ & 0.034 \\
\hline$\geq 4$, median (vs. $<4$ ) & $0.041(0.019)$ & 0.028 \\
\hline$\overline{\mathrm{Q}} 1: 0$ & (reference) & $<0.001^{\mathrm{T}}$ \\
\hline Q2: 1-2 & $0.025(0.027)$ & 0.36 \\
\hline Q3: 3-6 & $0.051(0.026)$ & 0.049 \\
\hline Q4: 7-32 & $0.094(0.028)$ & 0.001 \\
\hline
\end{tabular}

$\mathrm{BAI}=$ Beck Anxiety Inventory; BDI $=$ Beck Depression Inventory; $\mathrm{Q}=$ quartile; $\mathrm{T}=$ Trend; $\beta$ Est $=\beta$ estimate; $\mathrm{SE}=$ standard error of the mean.

sectional association between amyloid deposition and neuropsychiatric symptoms in presymptomatic $\mathrm{AD}$. For instance, in line with our findings, a systematic review observed significant differences in beta amyloid levels between depressed and nondepressed older adults in the majority of included studies (Harrington et al., 2015). In addition, the review concluded that individuals with depression showed higher amyloid binding on PET; however, the review only included five studies that measured beta amyloid using PET neuroimaging (two using $\mathrm{PiB}$, three using other ligands) (Harrington et al., 2015). Investigators from the Australian AIBL study recently reported that sex moderated the relationship between amyloid deposition, APOE genotype, and anxiety and depressive symptoms among 423 cognitively normal elderly persons (Holmes et al., 2016); whereas in our study, we did not observe an altering effect of sex on the association between anxiety and depressive symptoms and amyloid deposition. An Australian group also reported that anxiety significantly moderated the relationship between amyloid burden and decline in verbal and episodic memory among 178 subjects without dementia based on a cohort analysis (Pietrzak et al., 2014). They observed that among $\mathrm{PiB}+$ participants, those with increased anxiety symptoms had a significantly greater decline as compared to those without increased anxiety symptoms (Pietrzak et al., 2014). When the study was replicated one year later with a larger sample size of 333 participants, the researchers again observed that anxiety symptoms but not depressive symptoms moderated the effect of Aßrelated cognitive decline (Pietrzak et al., 2015). This is in line with our finding, as we observed a stronger association between amyloid deposition and anxiety as compared to the association between amyloid deposition and depression. Additionally, a study derived from the AD Neuroimaging Initiative (ADNI) database and involving MCI patients found that $A ß+$ subjects that presented with coexistent depressive symptoms had a higher risk of developing $\mathrm{AD}$ at a faster conversion rate than non-depressed counterparts. Moreover, the amount of deposited amyloid correlated with the rate of progression in depressed individuals (Brendel et al., 2015). In contrast to studies that reported a relationship between neuropsychiatric symptoms and amyloid deposition, a study among patients with $\mathrm{MCI}$ and $\mathrm{AD}$ failed to detect an association between cortical amyloid deposition and depressive symptoms (Chung et al., 2016). Our findings may be pertinent to the recently reported construct of mild behavioral impairment (MBI) which is defined as a change in behavior or personality in persons without dementia aged 50 years and older (Ismail et al., 2016). In our study, we observed an association between depressive and anxiety symptoms with cortical amyloid deposition. Some of the participants may have had a lifelong history of NPS. However, those that had new-onset NPS after the age of 50 can be classified as MBI; and these MBI subjects may 
have higher odds of having abnormal amyloid deposition in the brain. Furthermore, MBI subjects with elevated amyloid deposition may need to be closely followed in order to determine if they are at higher risk for developing incident dementia as compared to MBI subjects without cortical amyloid deposition. Previous research has shown that $\mathrm{PiB}$ retention may in part be mediated by $\mathrm{PiB}$ sulfation via estrogen sulfotransferase (Cole et al., 2010), an enzyme involved in brain inflammation. Therefore, one possible explanation of our study findings can be an association between depressive and anxiety symptoms and brain inflammation. However, future research is needed to investigate underlying mechanisms linking amyloid deposition with depressive and anxiety symptoms. Our findings should be interpreted in the context of the strengths and limitations of the study. Strengths of our study include the large scale, population-based sample size and the categorical and continuous assessment of depressive and anxiety symptoms. The main limitation of this analysis was the low number of participants with either depressive or anxiety symptoms, even though this was expected in this population-based study sample. This limited our power to detect associations between depressive and anxiety symptoms with elevated amyloid PET SUVR. Another limitation of the study is that both BAI and BDI-II are self-reported questionnaires, thus we cannot rule out recall bias. However, both assessments are widely used for clinical research purpose. Also, as in any cross-sectional study, the direction of causality between the association of amyloid deposition and depression and anxiety is unknown. In summary, we observed an informative albeit weak association between increased BDI and BAI scores and elevated cortical amyloid deposition. This observation needs to be tested by a longitudinal cohort study.

\section{Conflict of interest}

None.

\section{Description of authors' roles}

Formulation of the research question: KrellRoesch, Geda. Study design: Roberts, Mielke, Vemuri, Jack, Knopman, Boeve, Petersen, Geda. Conduct of the study: Lowe, Roberts, Mielke, Vemuri, Jack, Knopman, Boeve, Petersen, Geda. Data analysis: Christianson, Kremers. Drafting of manuscript: Krell-Roesch, Geda. Critical revision of manuscript: Lowe, Neureiter, Pink, Roberts,
Mielke, Vemuri, Stokin, Jack, Knopman, Boeve, Petersen.

\section{References}

Altman, D. G. and Royston, P. (2006). The cost of dichotomising continuous variables. BMF, 332, 1080.

Beck, A. T., Epstein, N., Brown, G. and Steer, R. A. (1988). An inventory for measuring clinical anxiety: psychometric properties. Fournal of Consulting and Clinical Psychology, 56, 893-897.

Beck, A. T., Steer, R. A. and Brown, G. K. (1996). BDI-II, Beck Depression Inventory: Manual. San Antonio, TX; Boston, MA: Psychological Corp.; Harcourt Brace.

Bjelland, I., Lie, S. A., Dahl, A. A., Mykletun, A., Stordal, E. and Kraemer, H. C. (2009). A dimensional versus a categorical approach to diagnosis: anxiety and depression in the HUNT 2 study. International fournal of Methods in Psychiatric Research, 18, 128-137.

Braak, H. and Braak, E. (1991). Neuropathological stageing of Alzheimer-related changes. Acta Neuropathologica, 82, 239-259.

Brendel, M., Pogarell, O., Xiong, G., Delker, A., Bartenstein, P. and Rominger, A. (2015). Depressive symptoms accelerate cognitive decline in amyloid-positive MCI patients. European fournal of Nuclear Medicine and Molecular Imaging, 42, 716-724.

Butters, M. A. et al. (2008). Imaging Alzheimer pathology in late-life depression with PET and Pittsburgh Compound-B. Alzheimer Disease and Associated Disorders, 22, 261-268.

Chetelat, G. et al. (2012). Relationship between memory performance and beta-amyloid deposition at different stages of Alzheimer's disease. Neurodegenerative Diseases, 10, 141-144.

Choi, S. H. et al. (2014). A three-dimensional human neural cell culture model of Alzheimer's disease. Nature, 515, 274-278.

Chung, J. K. et al. (2016). Cortical amyloid beta deposition and current depressive symptoms in Alzheimer disease and mild cognitive impairment. Fournal of Geriatric Psychiatry and Neurology, 29, 149-159.

Cole, G. B. et al. (2010). Specific estrogen sulfotransferase (SULT1E1) substrates and molecular imaging probe candidates. Proceedings of the National Academy of Sciences of the United States of America, 107, 6222-6227.

Donovan, N. J. et al. (2015). Depressive symptoms and biomarkers of Alzheimer's disease in cognitively normal older adults. Fournal of Alzheimer's Disease, 46, 63-73.

Dubois, B. et al. (2007). Research criteria for the diagnosis of Alzheimer's disease: revising the NINCDS-ADRDA criteria. Lancet Neurology, 6, 734-746.

Geda, Y. E. et al. (2006). Depression, apolipoprotein E genotype, and the incidence of mild cognitive impairment: a prospective cohort study. Archives of Neurology, 63, 435-440.

Geda, Y. E. et al. (2008). Prevalence of neuropsychiatric symptoms in mild cognitive impairment and normal cognitive aging: population-based study. Archives of General Psychiatry, 65, 1193-1198. 
Geda, Y. E. et al. (2014). Baseline neuropsychiatric symptoms and the risk of incident mild cognitive impairment: a population-based study. American fournal of Psychiatry, 171, 572-581.

Hardy, J. and Selkoe, D. J. (2002). The amyloid hypothesis of Alzheimer's disease: progress and problems on the road to therapeutics. Science, 297, 353-356.

Harrington, K. D. et al. (2017). Amyloid burden and incident depressive symptoms in cognitively normal older adults. International fournal of Geriatric Psychiatry, 32, 455-463.

Harrington, K. D., Lim, Y. Y., Gould, E. and Maruff, P. (2015). Amyloid-beta and depression in healthy older adults: a systematic review. Australian and New Zealand Fournal of Psychiatry, 49, 36-46.

Holmes, S. E. et al. (2016). beta-Amyloid, APOE and BDNF genotype, and depressive and anxiety symptoms in cognitively normal older women and men. The American Fournal of Geriatric Psychiatry, 24, 1191-1195.

Ismail, Z. et al. (2016). Neuropsychiatric symptoms as early manifestations of emergent dementia: provisional diagnostic criteria for mild behavioral impairment. Alzheimer's and Dementia, 12, 195-202.

Ivnik, R. J. et al. (1992b). Mayo's older americans normative studies: updated AVLT norms for ages 56 to 97 . Clinical Neuropsychologist, 6, 83-104.

Ivnik, R. J. et al. (1992c). Mayo's older Americans normative studies: WAIS-R norms for ages 56 to 97 . Clinical Neuropsychologist, 6, 1-30.

Ivnik, R. J. et al. (1992d). Mayo's older americans normative studies: WMS-R norms for ages 56 to 94 . Clinical Neuropsychologist, 6, 49-82.

Ivnik, R. et al. (1992a). Mayo's older americans normative studies: WAIS-R, WMS-R, and AVLT norms for ages 56 through 97. Clinical Neuropsychologist, 6, 1-104.

Jack, C. R. et al. (2010). Brain beta-amyloid measures and magnetic resonance imaging atrophy both predict time-to-progression from mild cognitive impairment to Alzheimer's disease. Brain, 133, 3336-3348.

Jack, C. R., Barrio, J. R. and Kepe, V. (2013). Cerebral amyloid PET imaging in Alzheimer's disease. Acta Neuropathologica, 126, 643-657.

Jagust, W. J. et al. (2010). The Alzheimer's disease neuroimaging initiative positron emission tomography core. Alzheimer's Eீ Dementia, 6, 221-229.

Knopman, D. S. et al. (2012). Short-term clinical outcomes for stages of NIA-AA preclinical Alzheimer disease. Neurology, 78, 1576-1582.

Krell-Roesch, J. et al. (2016). FDG-PET and neuropsychiatric symptoms among cognitively normal elderly persons: the mayo clinic study of aging. fournal of Alzheimer's disease, 53, 1609-1616.
Laborde-Lahoz, P. et al. (2014). Subsyndromal depression among older adults in the USA: prevalence, comorbidity, and risk for new-onset psychiatric disorders in late life. International fournal of Geriatric Psychiatry, 30, 677-685.

Malec, J. F. et al. (1992). Mayo's older americans normative studies: utility of corrections for age and education for the WAIS-R. Clinical Neuropsychologist, 6, 31-47.

Morris, J. C. et al. (2009). Pittsburgh compound B imaging and prediction of progression from cognitive normality to symptomatic Alzheimer disease. Archives of Neurology, 66, 1469-1475.

Murray, M. E. et al. (2015). Clinicopathologic and 11C-Pittsburgh compound B implications of Thal amyloid phase across the Alzheimer's disease spectrum. Brain, 138, 1370-1381.

Petersen, R. C. (2004). Mild cognitive impairment as a diagnostic entity. Fournal of Internal Medicine, 256, 183-194.

Petersen, R. C. et al. (2016). Association of elevated amyloid levels with cognition and biomarkers in cognitively normal people from the community. FAMA Neurology, 73, 85-92.

Pietrzak, R. H. et al. (2014). Anxiety symptoms, cerebral amyloid burden and memory decline in healthy older adults without dementia: 3-year prospective cohort study. British fournal of Psychiatry, 204, 400-401.

Pietrzak, R. H. et al. (2015). Amyloid-beta, anxiety, and cognitive decline in preclinical Alzheimer disease: a multicenter, prospective cohort study. $\mathscr{F}$ AMA Psychiatry, 72, 284-291.

Pink, A. et al. (2015). Neuropsychiatric symptoms, APOE epsilon4, and the risk of incident dementia: a population-based study. Neurology, 84, 935-943.

Pink, A. et al. (2017). Cortical thickness and anxiety symptoms among cognitively normal elderly persons: the mayo clinic study of aging. The fournal of Neuropsychiatry and Clinical Neurosciences, 29, 60-66.

Roberts, R. O. et al. (2008). The Mayo clinic study of aging: design and sampling, participation, baseline measures and sample characteristics. Neuroepidemiology, 30, 58-69.

Shaw, L. M., Korecka, M., Clark, C. M., Lee, V. M. and Trojanowski, J. Q. (2007). Biomarkers of neurodegeneration for diagnosis and monitoring therapeutics. Nature Reviews. Drug Discovery, 6, 295-303.

Sperling, R. A. et al. (2011). Toward defining the preclinical stages of Alzheimer's disease: recommendations from the national institute on aging-Alzheimer's association workgroups on diagnostic guidelines for Alzheimer's disease. Alzheimer's and Dementia, 7, 280-292.

Winblad, B. et al. (2004). Mild cognitive impairment-beyond controversies, towards a consensus: report of the international working group on mild cognitive impairment. Fournal of Internal Medicine, 256, 240-246. 